\title{
The Tricycle Mobile Robot Movement Simulation
}

\author{
Eugene V. Larkin ${ }^{1}$, Maxim A. Antonov ${ }^{1}$ and Alexander N. Privalov ${ }^{2}$ \\ ${ }^{1}$ Department of Robotics and Industry Mechanization, Tula State University, Tula, Russia \\ ${ }^{2}$ Tula State Lev Tolstoy Pedagogical University, Tula, Russia
}

\begin{abstract}
The analytical model of three wheels mobile robot as the control object is worked out with use of the theoretical mechanics. Model includes two parts, part which describes longitudinal acceleration and dynamics of maneuvers on the azimuth angle and part which describes transverse oscillations of trunk relatively to the ground. For description of acceleration characteristics and maneuvering on the azimuth angle robot is considered as the trunk with installed two direct current motors at the base of the isosceles triangle and absolutely passive wheel installed in the vertex of the isosceles triangle. For description of transverse oscillations robot is considered as rigid trunk placed on viscose-elastic supports, and every support moves on its own land trajectory. It is shown that the result obtained may be used both for direct synthesis of mobile robot control system, and for evaluation of Nyquist frequency of sensors signal digitizing, which minimize information losses in mobile robot digital control system.
\end{abstract}

\section{Introduction}

Tricycle mobile robots are of widely used due to their extremely simple design, structural sustainability of and simplicity of control actions [1,2]. In the case under investigation only two direct current electrical motors may be used both for longitudinal movement and for azimuth angle maneuvering, so control system may be two-loops one only. When tricycle moves on the terrain every wheel rolls on the track with specific individual occasional micro-profile. Trunk of the mobile robot, placed on the viscose-elastic supports, may be considered as mechanical filter for damping a road impacts. If mobile robot carries onboard equipment it is necessary to evaluate mechanical accelerations, which affect onto equipment after filtering of road noise on the stage of robot design. So for proper design and proper control of tricycle during the operation phase it is necessary to have the adequate mathematical model of robot space navigation $[3,4,5]$. Methods of analytical description of moving of vehicles in space are not widespread in an engineering practice, that explains relevance and urgency of investigations in this domain of mechanics of mobile robots.

\section{Kinematics of mobile robot}

Kinematics of mobile robot, as a transport vehicle, is shown on the Fig. $1[6,7]$, where $x O y z$ is the Earth coordinate system, placed into the center of mass $O$ of mobile robot trunk; $x$-coordinate is directed forward in the course of the robot movement; $z$-coordinate is directed vertically up; and $z$-coordinate complements the coordinate system to the right one. Robot is considered as the absolutely rigid trunk, based on viscose/elastic supports, placed at the vertex $A, B, C$ of isosceles triangle. For the simplicity assumption, that suspension points and center of mass are situated at the same plane, are made. Besides it is assumed that robot is symmetrical with respect to longitudinal vertical plane, passing through the center of mass of the trunk. Wheels $B$ and $C$ are active. Aggregated moment of this wheels provides robotlongitudinal movement, and difference moment provides turning on the course angle $\psi$. Wheel $A$ is strong passive. It provides third fulcrum only, and is installed on the support, which in turn rotates at hinge. Axis of rotation is perpendicular to the $A B C$-plane. The trunk has no longitudinal not transverse mobility degree relatively to supports, in such a way centers of wheels may move the straights line perpendicular ABC-plane only. Lengths of supports counterbalance the weight of trunk $M g$, where $M$ is the trunk mass; $g$ is the acceleration of gravity.

When robot is installed stock-still onto a horizontal surface supports $A, B, C$ have the initial lengths $S_{A}=S_{B}=S_{C}=S$, and the coordinates of suspension points $A, B, C$ and supporting points $A^{\prime}, B^{\prime}, C^{\prime}$ are as follows:

$$
\begin{aligned}
& \left(x_{A}, y_{A}, z_{A}\right)=\left(L_{A}, 0,0\right) \\
& \left(x_{C}, y_{C}, z_{C}\right)=\left(L_{B C}, \quad L, 0\right) \\
& \left(x_{B}, y_{B}, z_{B}\right)=\left(L_{B C}, L, 0\right) \text {; } \\
& \left(x_{A^{\prime}}, y_{A^{\prime}}, z_{A^{\prime}}\right)=\left(L_{A}, 0, S\right) \text {; } \\
& \left(x_{B^{\prime}}, y_{B^{\prime}}, z_{B^{\prime}}\right)=\left(L_{B C}, L, \quad S\right) \\
& \left(x_{C^{\prime}}, y_{C^{\prime}}, z_{C^{\prime}}\right)=\left(L_{B C}, \quad L, \quad S\right) \text {. }
\end{aligned}
$$


When robot moves the trunk spatial position is determined with both three linear co-ordinates, $x, y, z$, and three angles, such as the pitch angle $\vartheta$, the rolling angle $\gamma$ and the azimuth angle $\psi$.

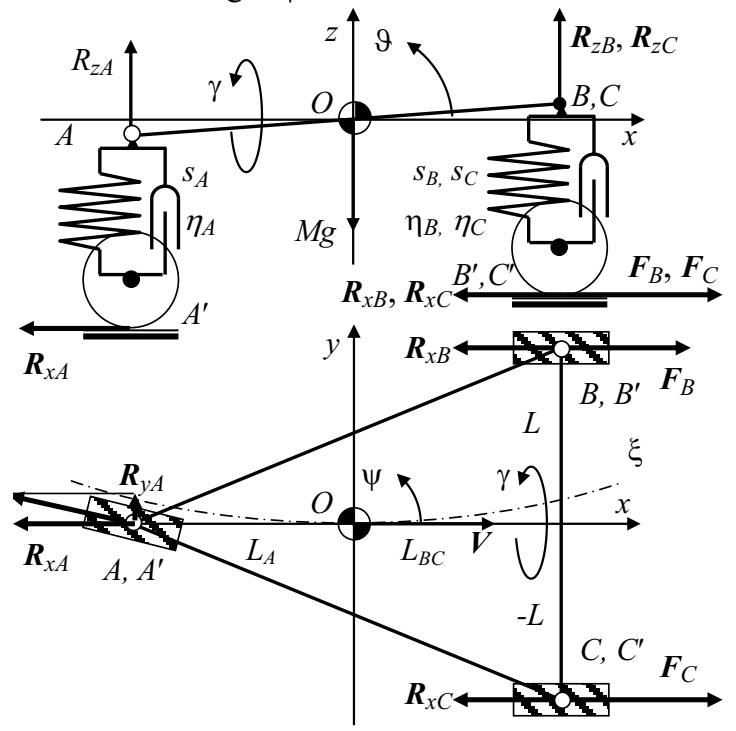

Figure 1. Kinematics of mobile robot

To describe robot movement one should to describe longitudinal motion and linked with it transverse oscillation of the trunk with respect to equilibrium position.

\section{Longitudinal movement of mobile robot}

Forces, which actuate the robot when longitudinal motion, are shown on the Fig $1[8,9,10,11]$. Designations of forces is as follows: $\xi$ is the trajectory of longitudinal movement; $\boldsymbol{V}, \dot{\psi}$ are longitudinal linear and transverse angular velocities of movement along the trajectory $\xi$, correspondingly; $\boldsymbol{F}_{B}, \boldsymbol{F}_{C}$ are vectors of driving forces in supports $B$ and $C$, correspondingly; $\boldsymbol{R}_{x A}, \boldsymbol{R}_{x B}, \boldsymbol{R}_{x} C$ are resistance forces, which include dry friction and rolling forces.

Concrete mathematical model of mechanical power source is determined with type of engine, used in concrete robot: it may be internal combustion engine, diesel engine or electrical motor [2, 3]. Simplest design has construction, based on two direct current motors, one of which actuates wheel $B$, and other actuates wheel $C$. For simplification of design motors are united with wheels. Control in such a system is divided onto $B$ and $C$ control subsystems.

For symmetrical robot differential equations, which describe control process in electrical engines, are as follows:

$$
\left\{\begin{array}{l}
T \dot{\omega}_{B}+\omega_{B}+K_{\mu} \mu_{B}+K_{v} v_{B}=K_{U} U_{B} \\
T \dot{\omega}_{C}+\omega_{C}+K_{\mu} \mu_{C}+K_{v} v_{C}=K_{U} U_{C},
\end{array}\right.
$$

where $\omega_{B}, \omega_{C}$ are rotation speeds of $B$ and $C$ motor shafts; $T$ is the time constant of motors; $\mu_{B}, \mu_{C}$ are payload moments on $B$ and $C$ motor shafts; $v_{B}, v_{C}$ are moments of dry friction in the bears of engine; $U_{B}, U_{C}$ are control actions applied to $B$ and $C$ motors; $K_{\mu}, K_{v}$, $K_{U}$ are proportionality factors;

If necessary, motors are equipped with reduction gears with transmitting coefficient $i$ (when motor have no reducer, then $i=1$ ). So moments and rotation speeds at reducer output shafts are as follows:

$$
\left\{\begin{array}{l}
\frac{r}{\mu_{B, C}}=\mu_{B, C} \\
r^{r} \omega_{B, C} i=\omega_{B, C}
\end{array}\right.
$$

where ${ }^{r} \omega_{B}{ }^{r} \omega_{C}$ are rotation speeds on reducer output shafts $B$ and $C$, respectively; ${ }^{r} \mu_{B},{ }^{r} \mu_{C}$ are payload moments on reducers $B$ and $C$ output shafts.

Reducers are loaded on the drive wheels, so wheel drive circumferential speeds, which coincide with the speeds of supports $B$ and $C$ are as follows:

$$
V_{B, C}={ }^{r} \omega_{B, C} q \text {. }
$$

Main parameter of vehicle, which characterizes its dynamic properties, is the accelerating parameter. To obtain it is sufficiently to work out the differential equation in small increments from a steady-state regime of movement (below increments with minus sign are decrements). Increments of a mass center longitudinal velocity $V$ and azimuth angle $\psi$ rotation speed are as follows:

$$
\left\{\begin{array}{l}
\delta_{V}=\frac{\delta_{V B}+\delta_{V C}}{2} \\
\delta_{\dot{\psi}}=\frac{\delta_{V B} \delta_{V C}}{2 L_{y}}
\end{array}\right.
$$

where $\delta_{V B}, \delta_{V C}$ are increments of circumferential speeds $V_{B}, V_{C}$ of $B$ and $C$ wheels; $q$ - is the radius of wheels; $\delta_{\dot{\psi}}$ is the increment of the azimuth rotation speed; $\dot{\psi}=\frac{d \psi}{d t}$.

Center of rotation is situated on the straight line passing through points $B$ and $C$ in parallel with y axis. The angle and the radius of rotation, which reckoned from the point, situated on the internal circumference, may be defined as follows:

$$
\psi_{\rho}=\frac{V_{C} d t-V_{B} d t}{2 L_{y}} ; \rho=\left\{\begin{array}{l}
\frac{2 L_{y} V_{B}}{V_{C}-V_{B}}, \text { when } V_{B}<V_{C} ; \\
\frac{2 L_{y} V_{C}}{V_{B}-V_{C}}, \text { when } V_{B}>V_{C},
\end{array}\right.
$$

where $d t$ is small increment of time.

Increments $\delta_{F B}, \delta_{F C}$ of driving forces $F_{B}, F_{C}$ on the wheels and increments $\delta_{r \mu B}, \delta_{r \mu C}$ of payload moments ${ }^{r} \mu_{B},{ }^{r} \mu_{C}$ are linked as follows:

$$
\delta_{F B, C} \cdot q=\delta_{r \mu B, C} \text {. }
$$


Differential equation system, which describes dynamic of longitudinal movement and azimuth angle rotation of mobile robot, is as follows:

$$
\begin{aligned}
& M \dot{\delta}_{V}+\eta_{V} \delta_{V}+\delta_{R x A}+\delta_{R x B}+\delta_{R x C}=\delta_{F B}+\delta_{F C} \\
& \quad J_{z} \dot{\delta}_{\dot{\psi}}+\eta_{\psi} \delta_{\dot{\psi}}+\delta_{R y A} L_{A}+\left(\delta_{R x B}-\delta_{R x C}\right) L_{y}= \\
& \quad=\left(\delta_{F B}-\delta_{F C}\right) L_{y}
\end{aligned}
$$

where $M$ is the summing mass of the trunk and supports; $J_{z}$ is the moment of inertia of the trunk with respect to axis $z ; \eta_{V}$ is the coefficient, which describes dissipative forces affects on the trunk (in the first approximation that force considered as viscous friction); $\eta_{\omega}$ is the coefficient, which describes dissipative forces in bears of wheels (they considered as moment of viscous friction too); $\delta_{V}$ is the increment of the longitudinal velocity $V$ of trunk; $\quad \dot{\delta}_{V}=\frac{d \delta_{V}}{d t} ; \delta_{R x A}$ is the increment of the longitudinal component of rolling and dry friction forces $R_{x A}$, affected on the wheel A; $\delta_{R y A}$ is the increment of the transverse component of rolling and dry friction forces $R_{y A}$ affected on the wheel $A ; \delta_{R x B}, \delta_{R x C}$ are increments of rolling and dry friction forces $R_{x B}, R_{x C}$, affected on the wheels $B, C ; \delta_{F B}, \delta_{F C}$ are increments of driving forces $F_{B}, F_{C}$ on wheels $B$ and $C$, correspondingly.

Joint solution of equations (3) - (9) gives following system, describing longitudinal movement and azimuth angle maneuvering of three wheels mobile robot:

$$
\delta_{V}(p) \cdot D_{V V}(p)+\delta_{\dot{\psi}}(p) \cdot D_{V \dot{\psi}}(p)=\delta_{F V}(p)
$$

$$
\delta_{V}(p) \cdot D_{\dot{\psi} V}(p)+\delta_{\dot{\psi}}(p) \cdot D_{\dot{\psi} \dot{\psi}}(p)=\delta_{F \dot{\psi}}(p),
$$

where $\mathrm{p}$ is the Laplace variable (differential operator);

$$
\begin{aligned}
& D_{V V}(p)=D_{\dot{\psi} V}(p)=p\left(M+\frac{2 T i^{2}}{K_{\mu} q^{2}}\right)+\left(\eta_{V}+\frac{2 i^{2}}{K_{\mu} q^{2}}\right) ; \\
& D_{V \dot{\psi}}(p)=-D_{\dot{\psi} \dot{\psi}}(p)= \\
& =p\left(\frac{J_{z}}{L_{y}}+\frac{2 T L_{y} i^{2}}{K_{\mu} q^{2}}\right)+\left(\frac{\eta_{\psi}}{L_{y}}+\frac{2 L_{y} i^{2}}{K_{\mu} q^{2}}\right) \text {; } \\
& \delta_{F V}(p)=\left(K_{U} \delta_{U B}-K_{v} \delta_{v B}\right) \frac{2 i}{K_{\mu} q}-\delta_{R x A}- \\
& -\frac{\delta_{R y A} L_{A}}{L_{y}}-2 \delta_{R x B} \text {; } \\
& \delta_{F V}(p)=\left(K_{U} \delta_{U C}-K_{V} \delta_{\nu C}\right) \frac{2 i}{K_{\mu} q}-\delta_{R x A}+ \\
& +\frac{\delta_{R y A} L_{A}}{L_{y}}-2 \delta_{R x C}
\end{aligned}
$$

$\delta_{U B}, \delta_{U C}$ are increments of control actions applied to $B$ and $C$ motors.

Solving the system (10) gives

$$
\delta_{V}(p)=\frac{\delta_{F V}(p) D_{\dot{\psi} \dot{\psi}}(p)-\delta_{F \dot{\psi}}(p) D_{V \dot{\psi}}(p)}{D_{V V}(p) D_{\dot{\psi} \dot{\psi}}(p)-D_{V \dot{\psi}}(p) D_{\dot{\psi} V}(p)} ;
$$

$$
\delta_{V}(p)=\frac{\delta_{F \dot{\psi}}(p) D_{V V}(p)-\delta_{F V}(p) D_{\dot{\psi} V}(p)}{D_{V V}(p) D_{\dot{\psi} \dot{\psi}}(p)-D_{V \dot{\psi}}(p) D_{\dot{\psi} V}(p)} .
$$

Expressions (11) shows, that dynamics of longitudinal movement of 3-wheel mobile robot depends on characteristics of motors, gear ratio, design of chassis etc. On the deviations of robot velocity changing load is influenced. It is necessary to admit, that (11) is valid, when wheels does not lose mechanical contact with a road and transverse slip of the wheels on the surface is absent

\section{Transverse oscillations of the trunk}

Transverse oscillations of trunk arise as a result of road impact, which are transmitted through viscose-elastic supports onto the trunk $[12,13,14,15]$. So coordinates of points of contact wheels and road are as follows

$$
\left(z_{A^{\prime}}, z_{B^{\prime}}, z_{C^{\prime}}\right)=\left(-S+\delta_{A},-S+\delta_{B},-S+\delta_{C}\right) .
$$

where $\delta_{A}, \delta_{B}, \delta_{C}$ are small deviations of supports length.

Trunk is the rigid, so for points $O, A, B, \mathrm{C}$ next condition is quite correct:

$$
\left|\begin{array}{llll}
x_{O} & y_{O} & z_{O} & 1 \\
x_{A} & y_{A} & z_{A} & 1 \\
x_{B} & y_{B} & z_{B} & 1 \\
x_{C} & y_{C} & z_{C} & 1
\end{array}\right|=0
$$

where $x_{O}, y_{O}, z_{O}$ are coordinates, which define spatial position of trunk mass center $O$; the $x_{A, B, C}, y_{A, B, C}, z_{A, B, C}$ are coordinates of points of mount of suspensions $A, B, C$, correspondingly.

As a rule, on the stage of mobile robot design one should only to evaluate the character of transverse oscillations of the trunk and effectiveness of those, or that design decisions. So the next assumption may be done for this purpose: all values, which characterize spatial position of the trunk are not much differ from their meaning at equilibrium position. In such a way below small increments $\delta_{z}, \delta_{\vartheta}, \delta_{\gamma}$ of the linear coordinate $z_{0}$, the pitch angle $\vartheta$ and the rolling angle $\gamma$, will be considered. Next formulae, which link increments $\delta_{A}, \delta_{B}, \delta_{C}$ and $\delta_{z}, \delta_{\vartheta}, \delta_{\gamma}$ may be obtained:

$$
\begin{gathered}
\delta_{z}=\frac{2 L_{B C} \delta_{A}+L_{A}\left(\delta_{B}+\delta_{C}\right)}{2\left(L_{A}+L_{B C}\right)} ; \\
\delta_{\vartheta}=\frac{\delta_{A}}{L_{A}+L_{B C}}-\frac{\delta_{B}}{2\left(L_{A}+L_{B C}\right)}-\frac{\delta_{C}}{2\left(L_{A}+L_{B C}\right)} ; \\
\delta_{\gamma}=\frac{\delta_{B}}{2 L}-\frac{\delta_{C}}{2 L} .
\end{gathered}
$$

Differential equations, which describe the trunk transverse oscillation, are as follows:

$$
\begin{gathered}
M \ddot{\delta}_{z}+\eta_{V} \dot{\delta}_{z}=\delta_{R z A}+\delta_{R z B}+\delta_{R z C} ; \\
J_{x} \ddot{\delta}_{\gamma}+\eta_{\omega} \dot{\delta}_{\gamma}=\left(\delta_{R z B}-\delta_{R z C}\right) L_{y} ; \\
J_{y} \ddot{\delta}_{\vartheta}+\eta_{\omega} \dot{\delta}_{\vartheta}=\delta_{R z A} L_{A}-\left(\delta_{R z B}+\delta_{R z C}\right) L_{B C}+ \\
+\left(\delta_{F B}+\delta_{F C}+\delta_{R x A}+\delta_{R x B}+\delta_{R x C}\right) S ; \\
\delta_{R z A, R z B, R z C}= \\
=\eta\left(\dot{\delta}_{A^{\prime}, B^{\prime}, C^{\prime}}-\dot{\delta}_{A, B, C}\right)+s\left(\delta_{A^{\prime}, B^{\prime}, C^{\prime}}-\delta_{A, B, C}\right),
\end{gathered}
$$


where $M$ is the mass of the trunk; $J_{x}, J_{y}$ are moments of inertia of the trunk with respect to $x$ and $y$ axis, correspondingly; $\eta_{V}, \eta_{\omega}$ are coefficients, which characterize dissipative forces affects on the trunk when linear and angle velocities correspondingly; (viscous friction); $\eta$ is the viscous friction factor, quite the same for dampers of supports $A, B, C ; s$ is the stiffness factor of support $A, B, C$ springs; $S$ is initial length of supports; $\delta_{z}, \delta_{\vartheta}, \delta_{\gamma}$ are increments of the linear coordinate $z_{0}$, the pitch angle $\vartheta$ and the rolling angle $\gamma$, correspondingly; $\dot{\delta}_{z, \vartheta, \gamma}=\frac{d \delta_{z, \vartheta, \gamma}}{d t} \quad ; \quad \ddot{\delta}_{z, \vartheta, \gamma}=\frac{d \dot{\delta}_{z, \vartheta, \gamma}}{d t} ;$ $\delta_{R z A}, \delta_{R z B}, \delta_{R z C}$ are increments of resistance forces $\boldsymbol{R}_{z A}, \quad \boldsymbol{R}_{z B}, \quad \boldsymbol{R}_{z C}, \quad$ correspondingly; $\quad \delta_{F B}, \delta_{F C}$ are increments of driving forces $\boldsymbol{F}_{B}, \boldsymbol{F}_{C}$; correspondingly; $\delta_{R x A}, \delta_{R x B}, \delta_{R x C}$ are increments of resistance forces

$\boldsymbol{R}_{x A}, \quad \boldsymbol{R}_{x B}, \quad \boldsymbol{R}_{x C}, \quad$ correspondingly; $\delta_{A}, \delta_{B}, \delta_{C}$ are increments of $z$ coordinates of suspension mount points $A$, $B, C ; \delta_{A^{\prime}}, \delta_{B^{\prime}}, \delta_{C^{\prime}}$ are increments of $z$ coordinates of points of touching of wheels $A, B, C$ the road; $\dot{\delta}_{A^{\prime}, B^{\prime}, C^{\prime}, A, B, C}=\frac{d \delta_{A^{\prime}, B^{\prime}, C^{\prime}, A, B, C}}{d t}$.

Equation system (15) is the linear one, so its Laplace transform is as follows:

$$
\begin{aligned}
& \left(M p^{2}+\eta_{V} p\right) \delta_{z}(p)=\delta_{R z A}(p)+\delta_{R z B}(p)+\delta_{R z C}(p) ; \\
& \left(J_{x} p^{2}+\eta_{\omega} p\right) \delta_{\gamma}(p)=\left[\delta_{R z B}(p)-\delta_{R z C}(p)\right] L_{y} ; \\
& \delta_{R z A, R z B, R z C}(p)=(\eta p+s)\left\lfloor\delta_{A^{\prime}, B^{\prime}, C^{\prime}}(p)-\delta_{A, B, C}(p)\right],
\end{aligned}
$$

where $\mathrm{p}$ is the Laplace variable (differential operator).

Solving (14), (16) with respect to $\delta_{z}, \delta_{\vartheta}, \delta_{\gamma}$ one would have the next dependences:

$$
\begin{gathered}
\delta_{z}(p)=W_{A \rightarrow z}(p) \cdot \delta_{A^{\prime}}(p)+W_{B \rightarrow z}(p) \cdot \delta_{B^{\prime}}(p)+ \\
W_{C \rightarrow z}(p) \cdot \delta_{C^{\prime}}(p)+W_{F B \rightarrow z}(p) \cdot \delta_{F B}(p)+ \\
+W_{F C \rightarrow z}(p) \cdot \delta_{F C}(p) ; \\
\delta_{\gamma}(p)=W_{B \rightarrow \gamma}(p) \cdot \delta_{B^{\prime}}(p)+W_{C \rightarrow \gamma}(p) \cdot \delta_{C^{\prime}}(p) ; \\
\delta_{\vartheta}(p)=W_{A \rightarrow \vartheta}(p) \cdot \delta_{A^{\prime}}(p)+W_{B \rightarrow \vartheta}(p) \cdot \delta_{B^{\prime}}(p)+ \\
W_{C \rightarrow \vartheta}(p) \cdot \delta_{C^{\prime}}(p)+W_{F B \rightarrow \vartheta}(p) \cdot \delta_{F B}(p)+ \\
+W_{F C \rightarrow \vartheta}(p) \cdot \delta_{F C}(p),
\end{gathered}
$$

where $W_{\alpha \rightarrow \beta}(p)$ transfer function, which describes reaction $\beta$ on the actuation $\alpha$.

Transfer functions have sixth order characteristic polynomial, what shows that there may be character frequencies, on which trunk is swinging at certain speeds of movement. To avoid swinging designer should to optimize design of robot, namely properly choice mass of the trunk and parameters of viscose-elastic supports.

\section{Result of computer simulation of mobile robot movement}

A result of simulation of movement of mobile robot is shown on the Fig. 2.

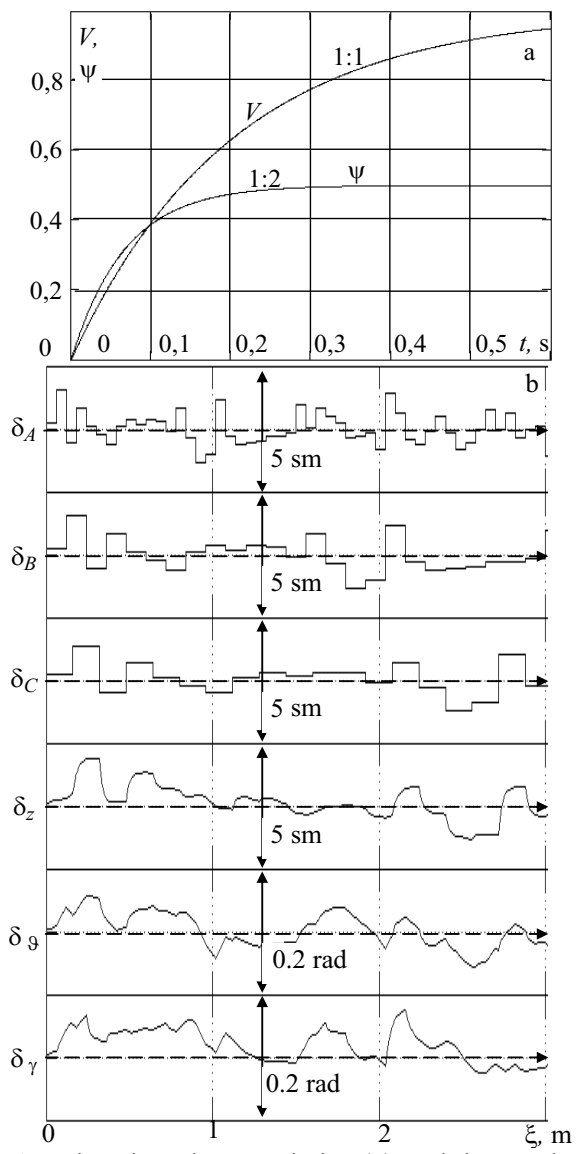

Figure 2. Acceleration characteristics (a) and the trunk transverse oscillations (b) of three wheels mobile robot

On the Fig. 2 a, acceleration characteristics for maneuvers along coordinate $\mathrm{x}$ and angle $\psi$ are shown in standardized intervals $0 \div 1$. For a convenience graphics are presented in different scales. As the input signal Haeviside function is used. For investigation of velocity dynamics step function was impacted on left and right control systems in-phase. For investigation of azimuth angle dynamics step function was impacted on left and right control systems counter-phase.

On the Fig. 2 b, transverse oscillations of trunk are shown. The case, when three wheels mobile robot moves on the terrain, and every wheel rolls on the track with specific individual micro-profile is considered. Road micro-profiles under wheels A, B, C were generated as "white", uncorrelated noises and are shown in the first three diagrams. Next three diagrams shows transverse oscillation on the linear coordinate $z_{0}$, the pitch angle $\vartheta$ and the rolling angle $\gamma$, correspondingly.

\section{Conclusion}

The result obtained may be used directly for synthesis of mobile robot control system, due to the fact, that all methods of synthesis used are based on the knowledge of control object dynamic model [16]. As a rule, mobile robot spatial position is controlled by Von Neumann type digital computer, which have plenty other tasks to solve. So there is the problem to divide computational resources of controller between solved tasks at a current time. One of parameters helping facilitation a solution of the 
problem is choice rational intervals between quests of sensors, in particular censors of spatial position of robot and speedometer. Quest time intervals should satisfy the sample theorem, and in particular frequency of quests should be more, then Nyquist frequency of sampling [17]. Mechanics of mobile robot may be considered as the filter, which smoothing impact of such disturbing factors, as billowy land. So model permits to evaluate sampling frequency, which minimize information losses when digitizing of sensors signal [18].

The research was carried out within the state assignment of the Ministry Education and Science of Russian Federation (No 2.3121.2017/PCH).

\section{References}

1. Mehmszow U. Mobile Robotics: A Practical Introduction. Springer-Verlag. London Ltg. 2003. $273 \mathrm{p}$.

2. Siciliano B., Khatib O. Springer Handbook of Robotics. Springer. 2016. 2155 p.

3. Sweet L. M., Strobel K. L. Dynamic models for control system design of integrated robot and drive systems //Journal of Dynamic Systems, Measurement, and Control. - 1985. - T. 107. - P. 53.

4. Sweet L., Good M. Redefinition of the robot motioncontrol problem //IEEE Control Systems Magazine. 1985. - Vol. 5. - №. 3. - Pp. 18 - 25.

5. Kamaldar M. et al. A control synthesis for reducing lateral oscillations of a spherical robot // ICM 2011. IEEE International Conference on Mechatronics. 2011. - Pp. 546 - 551.

6. Kurochkin S.A., Larkin E.V., Privalov A.N. Moving terrain objects simulators designing: Conception and methods of design // Курочкин С.А., Ларкин Е.В., Привалов А.Н. Проектирование тренажеров подвижных наземных объектов: Концепция и методология проектирования. - Saarbrucken Deutchland: LAP LAMBERT Academic Publishing GmbH \& Co., 2012. - 257 P. ISBN 978-3-84652687-3.

7. Dreizier R.M., Ludde C.S. Theoretical Mechanics. Theoretical Physics 1. Springer -Verlag. Berlin. Heidelberg. 2010. 402 p.
8. Janarthanan B., Padmanabhan C., Sujatha C. Longitudinal dynamics of a tracked vehicle: Simulation and experiment // Journal of Terramechanics. - 2012. - Vol. 49. - №. 2. - Pp. 63 72.

9. Ciobotaru T. Semi-empiric algorithm for assessment of the vehicle mobility // Leonardo Electronic Journal of Practices and Technologies. - 2009. - Vol. 8. - №. 15. - Pp. 19 - 30.

10. Bi X. et al. Transient modeling for heavy tracked vehicle performance and fuel consumption under accelerating conditions. - SAE Technical Paper, 2002. - №. 2002-01-0630.

11. Pacey D. A., Walker H. S. Modeling the dynamics of vehicles with differential-speed steering //Transactions of the ASAE. - 1996. - T. 39. - №. 2. - C. 431-434.

12. Chen L. Q. Analysis and control of transverse vibrations of axially moving strings // Applied Mechanics Reviews. - 2005. - Vol. 58. - №. 2. - Pp. 91-116.

13. Stépán G., Haller G. Quasiperiodic oscillations in robot dynamics // Nonlinear Dynamics. - 1995. - Vol. 8. - №. 4. - Pp. 513 - 528.

14. Hervas J. R., Reyhanoglu M., Tang H. Automatic landing control of unmanned aerial vehicles on moving platforms //Industrial Electronics (ISIE), 2014 IEEE 23rd International Symposium on. - IEEE, 2014. - C. 69-74.

15. H. Hu, E. Li, X. Zhao, Z. Liang and W. Yu, Modeling and simulation of folding-boom aerial platform vehicle based on the flexible multi-body dynamics // 2010 International Conference on Intelligent Control and Information Processing. Dalian. 2010. - Pp. 798 - 802.

16. Kilian C.T. Modern control technology: Components and systems. - Thompson Delmar Learning. 2000. $608 \mathrm{p}$.

17. Meyer-Baese U. Digital signal processing. SpringerVerlag Berlin, Heidelbrg, 2004. 523 p.

18. Babishin V., Taghipour S. Optimal maintenance policy for multicomponent systems with periodic and opportunistic inspections and preventive replacements //Applied Mathematical Modelling. 2016. - V. 40. -№. 23. - Рp. 10480 - 10505. 